\title{
A Cognitive Approach to the Detection of Spectrum Holes in Wireless Networks
}

\author{
Tobias Renk, Clemens Kloeck, and Friedrich K. Jondral
}

\begin{abstract}
The experience of ubiquitous and seamless access to heterogeneous mobile communication networks is one of the core issues of today's research. This comes along with an increasing demand in bandwidth. However, bandwidth as a natural resource is limited by technical constraints and, as several measurements have shown, is currently used very inefficiently due to a static allocation. Consequently, we have to consider spectrum allocation techniques and employ useful applications for the detection of vacant frequency bands. In this paper, we present a novel, swarm-behavior based approach for the detection of spectrum holes in cognitive wireless networks. It is based on the fact that several cognitive radios form a cognitive network. This network is then split up into several cognitive sub-networks that collaborate among each other and scan the frequency range simultaneously. Thus, several vacant frequency bands can be found and the overall processing time can be reduced. In addition, fading effects due to multi-path propagation can be met in a more efficient way.
\end{abstract}

\section{INTRODUCTION}

No one would question the fact that the demand for bandwidth - or data rate capacity - is dramatically rising in nower days and the near future. This can be seen with a glimpse on the enormous improvements in communication networks, e.g., the Internet. As a lot of frequency bands are already allocated in a very unflexible way, namely by selling access rights exclusively to users, it is very difficult to find free spectrum bands and to deploy new applications. Moreover, this resource is further limited by fundamental physical laws. However, measurements have shown that large parts of the spectrum lie idle during significant time periods. A fact that implies that spectrum is not really a scarce resource, but that it is used very inefficiently. Hence, new ways have to be found in order to allow a more dynamic and flexible allocation of frequency bands [1], [2]. One important issue in this context is the reliable detection of other users or, in other words, the reliable detection of spectrum holes. However, scanning the whole frequency range would be too time-wasting and power-consuming for a radio device.

This paper is organized as follows. Section II describes an appropriate radio concept in order to achieve seamless services in heterogeneous communication scenarios. In Section III a classification of spectrum is introduced and common spectrum sensing methods are explained. A novel, swarm-behavior based approach to the detection of

T. Renk, C. Kloeck, and F. K. Jondral are with the Institut für Nachrichtentechnik, Universität Karlsruhe (TH), Germany (email: \{renk, kloeck, fj\}@int.uni-karlsruhe.de) spectrum holes in wireless networks is shown in Section IV. Finally, Section V concludes the paper and summarizes the results.

\section{Cognitive Radio ConcePt}

In future mobile communication systems users should be able to enjoy seamless services in heterogeneous scenarios and ubiquitous access to networks wherever they are and whenever they want. This requires interworking between different radio access technologies, a condition that eventually demands for a smart radio resource management scheme. This can only be achieved by applying appropriate radio concepts. Such a concept of an intelligent radio is Cognitive Radio (CR) or, in other words, a brainempowered radio [3]. The term CR was firstly coined by J. Mitola III in 1999 [4] and established a perception towards new sophisticated procedures in radio communications. A CR is aware of its environment and has the ability to act accordingly to it due to its sensing, learning, and reasoning capabilities. In [3] three cognitive tasks are described. These are radio-scene analysis, which includes the detection of spectrum holes, channel identification, and transmit-power control and dynamic spectrum management.

\section{Detection of Spectrum Holes}

\section{A. Classification of Spectrum}

In [3] a spectrum hole is defined as a frequency band that is assigned to a primary user exclusively, but that is not allocated by this user at a specific time and place. Furthermore, spectrum can be classified into three types that indicate the amount of interference in a specific band:

- Black spaces: These spaces are highly occupied by local interferers.

- Grey spaces: These spaces are partially occupied.

- White spaces (spectrum holes): These spaces are free of local interferers. The only interference is due to ambient noise, e.g., thermal or impulsive noise.

This classification shows that black spaces are no proper candidates for dynamic spectrum allocation. However, grey spaces (to a certain amount) and white spaces can support dynamic allocation techniques. A crucial question at this point is how the detection of signals in noise and the previously described classification can be realized.

In Fig. 1 we see the spectrum occupation in the GSM 1800 downlink in a typical city of normal size from 19 o'clock to 20 o'clock. The different power levels on each 


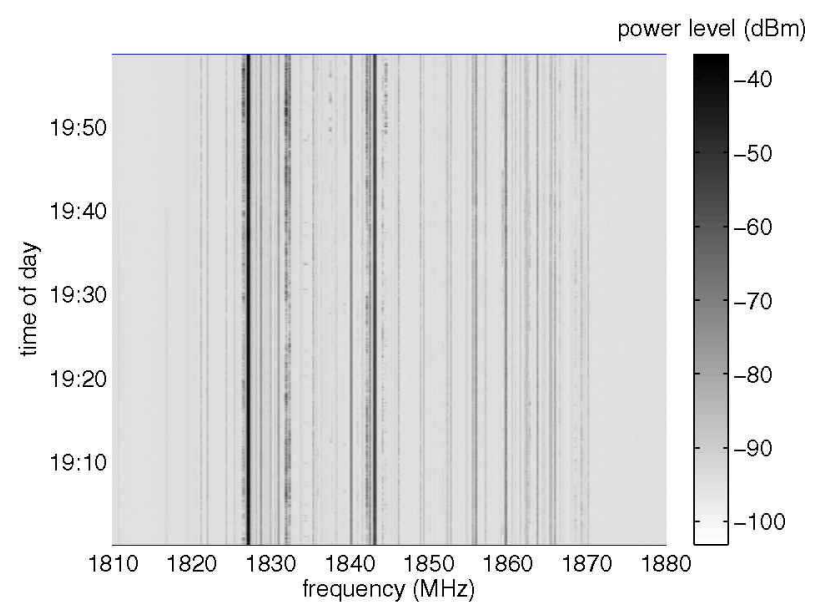

Fig. 1. Spectrogram that shows spectrum occupation in the downlink of a GSM 1800 system in a normal size city from 19 o'clock to 20 o'clock.

channel are represented through different color strengths where black indicates a highly occupied band.

\section{B. Interference Temperature}

The Spectrum Policy Task Force of the Federal Communications Commission proposed to use the sum of natural noise and interference noise in order to decide whether a transmitter is allowed to become active [3], [5]. For this purpose, a transmitter first has to measure the interference temperature, $T_{I}$, at the receiver. It is defined as follows:

$$
T_{I}\left(f_{c}, B\right)=\frac{P_{I}\left(f_{c}, B\right)}{k_{B} B},
$$

where $P_{I}\left(f_{c}, B\right)$ is the average interference power within the bandwidth $B$ around the center frequency $f_{c}$ and $k_{B}$ denotes the Boltzmann constant which is $1.38 \cdot 10^{-23} \mathrm{~J} / \mathrm{K}$. The value of interference temperature describes the power measured at a receiver per unit bandwidth. If it is below a given threshold, the transmitter will be allowed to send. If not, the transmitter will have to wait or simply change the frequency band. This approach makes sense as modern wireless communication systems consist of noise-like signals. Nevertheless, it is not really new.

\section{Matched Filter}

A matched filter can be characterized by the formula

$$
h(t)=\alpha \cdot s(T-t),
$$

where $s(t)$ is the transmit signal, $T$ is the symbol rate, and $\alpha$ is a factor that characterizes the amplitude. A matched filter maximizes the signal-to-noise ratio at the receiver [6]. Thus, it is the optimal choice for signal detection. One drawback of a matched filter is that it requires a priori knowledge of the transmitted signal for demodulation. This knowledge contains, e.g., the modulation scheme and the order of modulation. Additionally, time synchronization and even carrier synchronization are undispensable for coherent demodulation. This means for a CDMA system that the receiver must know the dedicated spreading codes and synchronization channels [7].

\section{Neyman-Pearson Criterion}

A well-known signal detection technique from radar systems is the Neyman-Pearson criterion. This criterion maximizes the detection probability at a given false alarm probability [8]. Consequently, both probabilities cannot be maximized together since they depend on each other. A proper value for the detection probability is $99.9 \%$ or even higher. However, such a high detection probability is difficult to achieve as the false alarm probability would be too high and degrade the overall system performance. A solution would be to enlarge the detection time to reduce the false alarm probability, but the system throughput would degrade. A diversity approach presented in [9] significantly improves the system performance. Diversity in this context means that not only one radio observes the spectral resource within a system, but all radios. This collaborative approach even allows to reduce the sensitivity requirements of each radio and to decrease the signal processing complexity [10], [11].

\section{E. Energy Detector}

A sub-optimal method for signal detection is energy detection that is known from radiometry [12]. After selecting the carrier frequency and the bandwidth of interest, the detected signal is squared and integrated in order to obtain the signal energy $E$ :

$$
E=\int_{t-T}^{t} y^{2}(\tau) d \tau
$$

Here, $E$ is the energy of the input signal $y(t)$ at any time over the interval $T$ in the past. The input signal $y(t)$ consists either of noise alone or a signal plus noise. Thus, detection means the test of the following hypothesis:

$$
\begin{aligned}
& H_{0}: y(t)=n(t) \\
& H_{1}: y(t)=s(t)+n(t)
\end{aligned}
$$

Afterwards, a free/used decision is made. Of course, processing gain can be improved as the observation time is increased. Another benefit of a longer observation time is the fact that the noise level is decreased, thus increasing the signal-to-noise ratio. In spite of these advantages, including a simple implementation, the energy detector owns some drawbacks. Since an energy detector is not able to differentiate between pure noise (thermal and background) and modulated signals, there is no possibility to cancel interferers by using signal processing techniques [6], [7].

\section{F. Cyclostationary based Detection}

Due to the fact that modulated signals are normally aligned with sine waves they bear some kind of periodicity. This means that their statistics can be described by cyclostationary processes, in spite of merely being stationary in nature. This cyclostationary can be exploited in the receiver for parameter estimation (e.g., pulse timing). This method allows the detection of specific modulated signals 
in an environments of other modulated signals and noise. Normally, the analysis of stationary signals is based on the autocorrelation function and the power spectral density. However, the power spectral density is a one-dimensional function of frequency. When cyclostationary behavior can be exploited, a related function named spectral correlation function can be expressed. In general, the spectral correlation function is a complex-valued, two-dimensional function [7].

\section{SWARm Intelligence Based SEnsor NETWORKS}

Nearly everyone has already wondered about flocks of birds that fly around in apparent perfect harmony and how those birds manage such a brilliant motion? The keyword is swarm intelligence [13]. The formation of single birds in a swarm helps everybody to take several advantages, e.g., at foraging. Here, every bird looks for food through the eyes of all the other birds. Thus, the probability of finding food is much higher. This example taken from fauna motivates our cognitive approach to the detection of spectrum holes where each CR represents a single individual of the group (network). Normally, sensor networks consist of relatively "dumb" sensors that send their information to a centralized entity. This centralized entity then performs explicit calculations. Our swarm-behavior based approach exploits further cooperation and communication among each sensor, thus, increasing the overall intelligence of the network.

Characteristics of swarm intelligence are, among others, flexibility which means adaptiveness to the environment and robustness against disturbances. In addition, there are several advantages, e.g., scalability, adaptation, autonomy, and parallelism [13], [14]. These advantages make swarm intelligence very attractive for technical issues, e.g., wireless ad-hoc networks.

\section{A. Collaborative Sub-networks}

We propose a fusion of several in the immediate vicinity located CRs to a cognitive network (swarm). The main goal of such a fusion is the quest for proper resources in order to built up communication abilities for the whole network. Afterwards, the network will be divided into several cognitive sub-networks each of them searching in a different region of the time-frequency-power landscape (Fig. 2). This approach is similar to the multipopulation approach described in [15]. It is obvious that closely located CRs should be merged into a cluster, thus, taking advantage of the cellular structure of mobile communication systems. Additionally, the introduction of the cognitive sub-network concept owns some outstanding benefits:

- Due to the fact that several sub-networks scan different parts of the whole frequency range, multiple vacant bands can be found.

- As the cognitive sub-networks work simultaneously the overall processing time can be reduced.

- Effects due to multi-path propagation can be met in a more efficient way.

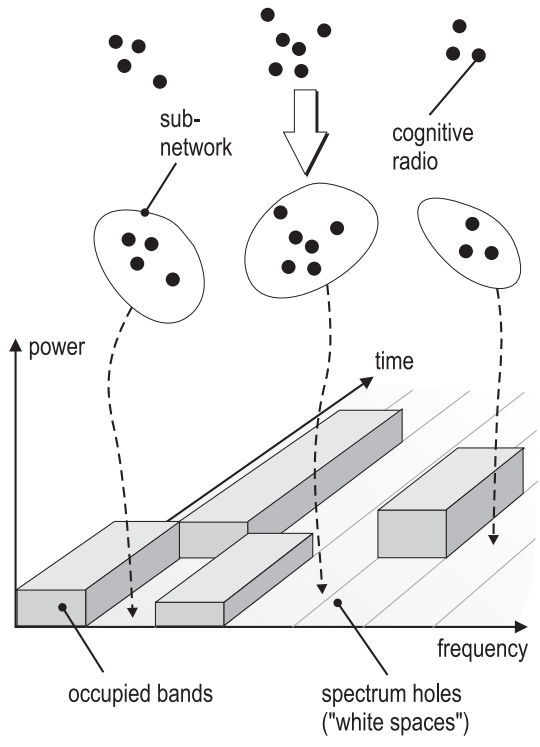

Fig. 2. Possible scenario description of some cognitive radios that group to clusters and search spectrum holes in cooperation.

The search within a sub-network is based on either Particle Swarm Optimization (PSO) or Evolutionary Algorithms (EAs) [15]. PSO is an iterative, population-based, stochastic optimization technique that is inspired by the social behavior of animals. It shares many similarities with EAs, however, in contrast to EAs, PSO has no evolution components like reproduction or mutation. PSO consists of a swarm of particles flying through the search space. Assigned to our approach the swarm of particles stands for a cognitive sub-network and the search space is presented by the time-frequency-power landscape (fitness landscape) where a small value of the fitness landscape indicates that a specific frequency band is not allocated by any other user yet. Each particle $i$ owns a position, $\mathbf{x}_{i}$, and a velocity, $\mathbf{v}_{i}$, that are iteratively updated. The movement through the search space is attracted by two "best" values, namely the personal best value, $\hat{\mathbf{x}}_{i}$, (the best solution a particle has achieved so far) and the local best value, $\hat{\mathbf{x}}_{l}$, (the best solution of any particle in the neighborhood). If a particle takes all the population in a swarm as its topological neighbors, the best value is called global best, $\hat{\mathbf{x}}_{g}$. At each iteration the position and the velocity are updated accordingly to the following equations:

$$
\begin{aligned}
& \mathbf{x}_{i}=\mathbf{x}_{i}+\mathbf{v}_{i} \\
& \mathbf{v}_{i}=\psi\left[\mathbf{v}_{i}+c_{1} \mathbf{r}_{1}\left(\hat{\mathbf{x}}_{i}-\mathbf{x}_{i}\right)+c_{2} \mathbf{r}_{2}\left(\hat{\mathbf{x}}_{l}-\mathbf{x}_{i}\right)\right]
\end{aligned}
$$

The parameter $\psi<1$ denotes an inertial constant that slows down the particles, thus allowing a better exploration. The constants $c_{1}$ and $c_{2}$ define the attraction towards neighboring particles, whereas $\mathbf{r}_{1}$ and $\mathbf{r}_{2}$ are vectors of uniformly distributed variables in the range $[0,1]$.

One remaining problem is a possible collision of different sub-networks even if they start their search in different areas of the search space. Under certain circumstances, it may happen that two sub-networks try to allocate the same frequency band. To avoid this the principle 
of exclusion is introduced in [15]. Exclusion means that if two sub-networks come too close each other (the distance between two sub-networks is the distance between their global bests) they compete and the one with the lower fitness will be re-initialized.

Most real-world problems are dynamic. This means in our context that the frequency allocation will change in time. In other words, the shape of our fitness landscape will change. One possibility to consider this behavior is the randomly reinitialization of all sub-networks everytime a change of the landscape occurs. However, this approach is too time-consuming and would reduce the probability for a successful search. Hence, knowledge of former search results should be somehow taken under consideration in order to define a new starting point for searching.

\section{B. Initialization Problem and Utilization Function}

One of the most difficult issues that has to be solved is the aspect of initialization. We have to bear in mind that idle spectrum resources depend on frequency, time, and location. As a consequence, initialization makes a great contribution to the reliable and successful detection of spectrum holes. A reasonable initialization can, for example, abbreviate the search period enormously. In contrast to that, a bad initialization can lead to a situation in which a communication cannot be set up properly within a predefined waiting period. The crucial question that has to be solved first can be expressed like this: How can we divide a network consisting of several CRs into several subnetworks such that the search for white spaces can be optimized? For the sake of simplicity we will first concentrate on the parameter frequency and ignore the factors time and location. Consider a set $\mathcal{M}=\{1,2, \ldots, M\}$ that consists of $M$ independent CRs. We now have to look for a proper subset $\mathcal{L}$ of the power set of $\mathcal{M}, \mathbf{P}(\mathcal{M})$, that suits best the current frequency situation:

$$
\mathcal{L} \subseteq \mathbf{P}(\mathcal{M})
$$

In addition, we assume that each $\mathrm{CR}$ can only belong to one sub-network. If we express the elements of a proper solution of (8) with $l$, this yields

$$
l_{i} \cap l_{j}=\emptyset \quad \forall l_{i}, l_{j} \in \mathcal{L} \quad \wedge \quad i \neq j,
$$

which simply means that all sets of $\mathcal{L}$ must be disjoint. For a first approach we take the spectral proximity between different CRs as similarity measure (a more precise description of this measure is given in section IV-C). To do so, a kind of master for a particular sub-network has to be defined. This is done by a radio device transmitting a beacon that can be received by everyone in the near proximity and which indicates that the sender claims to be the master. However, at the moment we have no knowledge about the optimal number of subsets, $|\mathcal{L}|$, and the optimal number of CRs in a subset, $\left|l_{i}\right|$.

To be able to evaluate solutions, we need a utilization function $u^{(k)}, k=1,2, \ldots, K$, where $k$ denotes a spe- cific sub-network and $K$ is the maximum number of subnetworks. We define a set

$$
\mathcal{Q}^{(k)} \triangleq\left\{\operatorname{QoS}^{(k, 1)}, \operatorname{QoS}^{(k, 2)}, \ldots, \operatorname{QoS}^{(k, N)}\right\}, N<M
$$

whose entries describe the difference between the required Quality of Service (QoS) of each CR and the obtained one within the $k$-th sub-network that consists of $N$ cognitive devices (the expression QoS contains parameters like data rate, bandwidth, bit error rate, signal-to-noise ratio, ...). Thus, we get

$$
\operatorname{QoS}^{(k, n)}=\left(\operatorname{QoS}_{\text {req }}^{(k, n)}-\operatorname{QoS}_{\mathrm{obt}}^{(k, n)}\right)^{+} \forall n=1, \ldots, N,
$$

where $\left(\operatorname{QoS}_{\text {req }}^{(k, n)}-\operatorname{QoS}_{\mathrm{obt}}^{(k, n)}\right)^{+}=\operatorname{QoS}_{\text {req }}^{(k, n)}-\mathrm{QoS}_{\mathrm{obt}}^{(k, n)}$ if $\operatorname{QoS}_{\mathrm{req}}^{(k, n)}>\operatorname{QoS}_{\mathrm{obt}}^{(k, n)}$ and 0 otherwise. The utilization function can then be defined as follows:

$$
\begin{aligned}
u^{(k)}= & \sum_{n=1}^{N}\left(\operatorname{QoS}_{\text {req }}^{(k, n)}-\mathrm{QoS}_{\mathrm{obt}}^{(k, n)}\right)^{+} \\
= & \sum_{n=1}^{N}\left(\operatorname{QoS}_{\mathrm{req}}^{(k, n)}-\mathrm{QoS}_{\mathrm{obt}}^{(k, n)}\right) \\
& \cdot \mathbf{1}\left[\left(\mathrm{QoS}_{\mathrm{req}}^{(k, n)}-\mathrm{QoS}_{\mathrm{obt}}^{(k, n)}\right)>0\right]
\end{aligned}
$$

The overall aim is the minimization of $u^{(k)}$ :

$$
\min u^{(k)} \quad \forall k=1,2, \ldots, K
$$

The utilization function expresses the fact that a CR that obtains more QoS than required will have no additional benefits from it as it is not able to use the extra resources adequately. This results in an overall benefit of each cognitive sub-network and, consequently, in the overall benefit of the whole network. However, as already indicated, this is a very challenging task that is under research investigation at the moment.

\section{Acceptance and Blocking Behavior}

Other questions we are faced with are the following: How can a CR that switches on be integrated into a consisting sub-network? How does it know which sub-network suits best in order to built up a reliable communication? To answer these questions we have to bear in mind that CRs that are already members of a sub-network should not suffer from a new arriving device too much.

Let the difference between the frequency of the master of the $k$-th sub-network, $f_{\text {mas }}^{(k)}$, and the new $\mathrm{CR}, f_{\mathrm{CR}}$, be

$$
\Delta f^{(k)}=\left|f_{\mathrm{mas}}^{(k)}-f_{\mathrm{CR}}\right|, \quad 1 \leq k \leq K
$$

The users already attending a sub-network may suffer a QoS degradation, $\Delta \mathrm{QOS}^{(k)}$, when a new user $i$ enters [16]:

$$
\Delta \operatorname{QoS}^{(k)}=\sum_{n=1}^{N} \operatorname{QoS}^{(k, n)}-\sum_{n=1, n \neq i}^{N} \widehat{\operatorname{QOS}}^{(k, n)}
$$




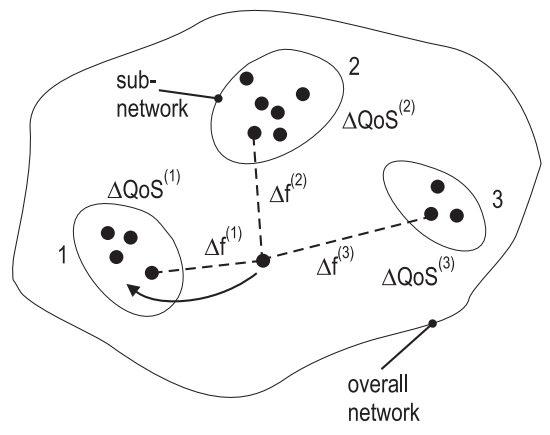

Fig. 3. Schematic illustration of the analysis process of the proximity set $\mathcal{P}^{(k)}, k=1,2,3$, where a new cognitive radio decides to join subnetwork 1 .

$\sum_{n=1}^{N} \operatorname{QoS}^{(k, n)}$ describes the QoS before the new CR requests and $\sum_{n=1, n \neq i}^{N} \widehat{\mathrm{QOS}}^{(k, n)}$ is the sum of the QoS for all users but the new one after its attendance to the subnetwork. Now, we are able to define the proximity function

$$
p^{(k)}=\frac{\Delta f^{(k)}}{1-\left(\Delta \operatorname{QoS}^{(k)} / \sum_{n=1}^{N} \operatorname{QoS}^{(k, n)}\right)},
$$

which indicates the similarity of a CR to the sub-network $k$. The denominator is designed such that it adopts values in $[0,1]$. The proximity functions for the different $K$ subnetworks are stored in the proximity set

$$
\mathcal{P}^{(k)} \triangleq\left\{p^{(1)}, p^{(2)}, \ldots, p^{(K)}\right\}, \quad K<M .
$$

All a new CR now has to do is to find the minimal value of this set:

$$
\min _{1 \leq k \leq K} \mathcal{P}^{(k)}
$$

For example, if $p^{(1)}<p^{(j)}$, where $2 \leq j \leq K$, then the CR moves to the sub-network 1 if the QoS degradation for the other CRs is not too severe. A special case occurs if $\Delta \operatorname{QoS}^{(k)} / \sum_{n=1}^{N} \operatorname{QoS}^{(k, n)}=1$, which indicates that the QoS requirements in the $k$-th sub-network will entirely break down. As a consequence, $p^{(k)} \rightarrow \infty$ and the $\mathrm{CR}$ can never be a member of this sub-network. On the other hand, if $\Delta \mathrm{QoS}^{(k)} / \sum_{n=1}^{N} \mathrm{QoS}^{(k, n)}=0$, the subnetwork will not suffer any QoS degradation when a new $\mathrm{CR}$ enters. Hence, the only remaining factor describing the proximity is $\Delta f^{(k)}$. Another special case is the one where $\Delta f^{(k)}=0$. This means indeed that $p^{(k)}$ turns to 0 , but we have to bear in mind that this situation might cause severe interferences to the master device and should be avoided. The whole process of acceptance is illustrated schematically in Fig. 3.

\section{CONCLUding REMARKS}

In this paper we presented a cognitive, swarm-behavior based approach to the detection of spectrum holes in wireless networks. After having introduced an appropriate radio concept, we explored the idea of using the interference temperature as criterion for transmission. Moreover, an overview of common detection techniques including matched filter, Neyman-Pearson criterion, energy detector, and detection based on cyclostationary processes was given. Our approach forms a network of CRs that is split up into several sub-networks. Each sub-network then searches for spectrum holes in a different area of the search space. Due to this distribution the overall processing time will be dramatically reduced. Another benefit is the ability to discover multiple minima in the time-frequency-power landscape. Furthermore, we faced the problem of initialization and introduced a suitable utilization function to evaluate the performance of the sub-networks. Finally, we presented measures to describe the acceptance and blocking behavior.

\section{ACKNOWLEDGMENT}

This work has been performed in the framework of the EU funded project End-to-End Reconfigurability $\left(E^{2} R\right)$. The authors would like to acknowledge the contributions of their colleagues from $\mathrm{E}^{2} \mathrm{R}$ consortium.

\section{REFERENCES}

[1] Q. Bi, G. I. Zysman, and H. Menkes, "Wireless Mobile Communications at the Start of the 21st Century," IEEE Communications Magazine, January 2001, pp. 110-116.

[2] R. Berezdivin, R. Breinig, and R. Topp, "Next-Generation Wireless Communications Concepts and Technologies," IEEE Communications Magazine, vol. 40, no. 3, March 2002, pp. 108-116.

[3] S. Haykin, "Cognitive Radio: Brain-Empowered Wireless Communications," IEEE Journal on Selected Areas in Communications, vol. 23, no. 2, February 2005, pp. 201-220.

[4] J. Mitola III, "Cognitive Radio: An integrated agent architecture for software defined radio," Dissertation, Computer Communication System Laboratory, Department of Teleinformatics, Royal Institute of Technology (KTH), Stockholm, Sweden, 2000.

[5] Federal Communications Commission, "Spectrum Policy Task Force Report," ET Docket No. 02-135, November 2002

[6] A. Ghasemi, E. S. Sousa, "Collaborative Spectrum Sensing for Opportunistic Access in Fading Environments," Proc. First IEEE International Symposium on New Frontiers in Dynamic Spectrum Access Networks (DySPAN 05), Baltimore, November 2005.

[7] D. Cabric, S. M. Mishra, and R. W. Brodersen, "Implementation Issues in Spectrum Sensing for Cognitive Radios," Asilomar Conference on Signals, Systems, and Computers, 2004.

[8] J. Hillenbrand, T. Weiss, and F. K. Jondral, "Calculation of detection and false alarm probabilities in spectrum pooling systems," IEEE Communications Letters, vol. 9, no. 4, April 2005, pp. 349351.

[9] T. Weiss, J. Hillenbrand, and F. K. Jondral, "A Diversity Approach for the Detection of Idle Spectral Resources in Spectrum Pooling Systems," Proc. of the 48th Int. Scientific Colloquium, Ilmenau, Germany, 2003.

[10] E. Visotsky, S. Kuffner, and R. Peterson, "On Collaborative Detection of TV Transmissions in Support of Dynamic Spectrum Sharing," Proc. First IEEE International Symposium on New Frontiers in Dynamic Spectrum Access Networks (DySPAN 05), Baltimore, November 2005, pp. 338-343.

[11] S. M. Mishra, A. Sahai, and R. Brodersen, "Cooperative Sensing among Cognitive Radios," IEEE International Conference on Communications (ICC 06), Istanbul, June 2006.

[12] H. Urkowitz, "Energy detection of unknown deterministic signals," Proceedings of IEEE, vol. 55, April 1967, pp. 523-531.

[13] E. Bonabeau, G. Theraulaz, and M. Dorigo, "Swarm intelligence: From Natural to Artificial Systems," Oxford University Press, New York, 1999.

[14] I. Kassabalidis, M. A. El-Sharkawi, R. J. Marks II, P. Arabshahi, and A. A. Gray, "Swarm Intelligence for Routing in Communication Networks," IEEE Global Telecommunications Conference (GLOBECOM 01), vol. 6, San Antonio, Texas, November 2001, pp. 3613-3617.

[15] T. Blackwell, J. Branke, "Multi-Swarms, Exclusion, and AntiConvergence in Dynamic Environments," IEEE Transactions on Evolutionary Computation, 2006.

[16] V. Krishna, "Auction Theory," Academic Press, San Diego, California, 2002. 